\title{
IS INSANITY A DISEASE OF THE MIND, OR OF THE BODY?
}

The Dependence of Insanity on Physical Disease, by Jous P. GrAY, M. D., Superintendent of the New York State Lunatic Asylum. [Read before the Medical Society of the State of New York, at its annual meeting, February 7, 1871.] American .Tournal of Insanity, April, 1871.

Materialism in its Relations to the Causes, Conditions and Treatment of Insanity, by H. B. WirbUR, M. D., Superintendent of the New York Asylum of Idiots. [A paper read before the Association of Superintendents of Insane Asylums, at the last annual meeting, at Toronto.] Journal of Physiological Medicine, January, 1872.

The papers here cited present two opposite views held by pathologists as to the nature, causes and treat. ment of insanity. On the one hand it is held to be a disease of the brain, originating in that organ or in other parts of the body, depending on physical causes, or on moral causes only so far as they are capable of producing physical effects, and to be treated by the same methods as other bodily diseases. On the other hand it is held to be a disease of the immaterial mind, depending for the most part directly on moral causes, and to be treated mainly by moral agencies. The object of this paper is to define as clearly as possible the views of these two schools of pathologists and discuss some of the problems involved.

I.

That a certain relation of mutual dependence exists between the mind and the brain is generally admitted by metaphysicians as well as by physiologists; but 
while the former occupying themselves exclusively with the phenomena of consciousness lose sight of their dependence on the brain, the latter, engaged in the study of physical structure and material movements, lose sight of mind, and confound mental phenomena with cerebral movements. It becomes then of some importance to establish the distinction between these two kinds of phenomena, and to define the relation they bear to each other.

By means of the five senses we observe certain things and phenomena, which together make up the whole external world known to us. The study of these things, their description and classification, the analysis of these phenomena, and the determination of the laws regulat. ing their order of succession, constitute the matter of physical science.

There are other phenomena which we do not find by the five senses, but by a faculty called consciousness, which we do not refer to the external world but to our own selves, as occurring in us. Thus, I know that I am now thinking on a certain subject and that certain thoughts succeed each other within me. How do I know this? I can not see nor hear myself think; though all my senses were abolished I might still think and know that I was thinking. I can only say that just as $I$ hear and feel and see the things and phenomena around me, so I am conscious of thought, emotion and will within me. I an even more sure of this latter knowledge than of the former, for my senses have often deceived me, and I receive their testimony with some distrust; but when I am conscious that I feel or think or will, I am sure I am not mistaken. I may admit that my thoughts are erroneous, that my feelings are unworthy or my volitions criminal, yet it would be absurd for any one to try to convince me that I am not think. 
ing, or feeling, or willing, when my consciousness tells me that I am.

Here then are facts of which we are most sure, which are not cognizable by the senses, but by consciousness; which are not movements or changes in the external world, but which occur within ourselves; and these facts may be observed, studied and reduced to a science. As out of the materials furnished by the senses, the physical sciences are constituted, so out of the facts of consciousness is constituted the science of Psychology.

The facts furnished by the senses have relation to the extended, moving thing we call matter, and so physical science is the science of matter. A fact of consciousness, such as thought, emotion or will, has no relation to extension nor figure, nor movement in space, nor any other material attribute, and hence we say it does not belong to matter, it is immaterial. As we name the substance having the attributes of extension and motion, matter, so we name the substance to which belong the attributes of thought, feeling and will, mind, and Psychol. ogy is the science of mind.

We have then these two great divisions of human knowledge, into Physical Science and Psychology.

If, having established this distinction, we stop short and consider these two divisions of science as having no relation to each other, we fall into a grave error, and form a very inadequate idea of Psychology. The phenomena of consciousness are connected with certain molecular movements of the brain, and dependent on them, and although these movements are not the phenomena of consciousness they are necessary factors in their production.

Physiology has demonstrated that mental phenomena are connected with molecular movements of the brain, so that during every thought, emotion or volition, move- 
ments in the cerebral hemispheres parallel to these, but distinct from them, go on. A blow on the head may suspend these movements, and with them the accompanying mental operation; a torpid circulation through the cerebral capillaries, or a depraved condition of the blood, impedes them; disease of the brain perverts them. What is the exact nature of these material conditions accompanying mental phenomena? What is their exact seat in the hemispheres? Physiology gives as yet no satisfactory answer to these questions; it has only established the existence of these movements. When a man is angry or frightened, a molecular cerebral change occurs, which certainly is not anger or fright, but the necessary material condition of such emotions; and if the science of the physiologist were sufficiently advanced, and all the parts could be exposed to his observation, he might interpret this molecular movement, and know that anger or fright existed in the mind of the person; just as a telegraphic operator, hearing the click of his instrument, knows the message it is carrying.

The relation which the cerebral movement bears to consciousness, presents the most obscure and difficult subject of human speculation, and all efforts to form any adequate conception of it are vain. The progress of physiology may hereafter demonstrate more clearly the nature of the nervous movement, and the seat of it cor: responding to various mental conditions; but whatever advance may be made in this direction, there must al. ways remain between the physiological and the psychological phenomenon an immense gap which we can not fill, even with a plausible hypothesis.

As far as our experience goes, mind, in a state of activity, never exists without a living brain. As to the mode of existence of the soul after death, we can know nothing by experience, nor can we draw any deduction 
by reasoning. The Christian Church teaches as a fundamental article of the creed, "the resurrection of the body ;" and although different interpretations have been put on this, yet it seems, at least, to point to the continuance hereafter of some relation between the soul and body, like that now existing.

Some physiologists, occupied with the study of physical phenomena, using their senses more than their consciousness, see only the cerebral movements accompanying mental operations, and neglect the latter or confound them with the former. Thought, feeling, and will, are by them called functions, or products of the brain, just as secretion is a product of glands; and hence the existence of mind is denied, and psychology becomes for them only a chapter of physiology. These are called Materialists.

This view is weaker than that of those psychologists who overlook the action of the brain accompanying mental phenomena; for it is possible to construct a science out of the facts of consciousness alone, but it is not possible to take the first step in mental science, if we reject the data of consciousness. No possible study of the brain could suggest the idea of anger or fear, if we had never felt these emotions in our consciousness. We might learn the nervous movements accompanying them, but these are not anger or fear, any more than the click of the telegraph is the intelligence it transmits. When I read of thought or emotion as a secretion or product of the brain, the words convey to me no idea whatever. It is as though one should say that thought is a triangle. I can conceive of molecular movements of the brain as giving rise to other movements, or as generating products, having extension and mobility, in short, some form of matter; but thought is a phenomenon occurring in myself and not in my body; it is not a material 
thing, nor has it the attributes of matter, it is not a product of the brain, though the brain may be necessary for its production.*

The preceding statement was necessary before entering on the main questions discussed in the papers, because their solution must depend on the views adopted of the relation of the mind and mental operations to the brain and cerebral movements. Dr. Gray advocates the views which have been here put forth, fortifying his position by extracts from writers on the subject, and expressly discarding the doctrine that the mind is a "mere result of cerebral action, and thus a material substance, a mere secretion liable to disease and death." It is to be regretted that Dr. Wilbur should have entitled his paper, which is mainly occupied in contesting the views of Dr. Gray, "Materialism in its Relations, \&c.," as if the latter were a materialist; for this word is now deservedly odious, as representing a doctrine weak in science and unsound in morals. He does indeed say that Dr. Gray has avowed himself to be no materialist, but intimates that this avowal is not consistent with his pathology of insanity.

It is not easy from his paper to arrive at Dr. Wil. bur's views on this point. He believes in the existence of mind as something apart from the body, and also in the reciprocal influence of the mind and body, but seems to confine this influence within very narrow limits.

\footnotetext{
* If we would speculate on this problem, which we can not solve, instead of considering the mind as a product of the brain, it may better be maintained that the brain is a product of the mind, which has formed this organ as a means of communicating with the external world, acting at first unconsciously as the plastic force presiding over the growth and development of the body, and when it has formed the nervous system, attaining to knowledge of the external world and to self-consciousness.
} 
He says, page 42, as expressing his own views: "The brain was regarded as the organ of mind, the instrument through which the sensational basis of thought or mental action was received, one remove nearer the thinking ego than the nerves of sensation and the organ of mind in fulfilling its purposes; behind in course and action the nerves of voluntary motion. Like the other and subsidiary portions of the nervous system it is sub. ject to the laws, conditions and effects of agency."

The meaning of this passage is not clear. In considing "the brain as the instrument through which the sens. ational basis of thought and mental action is received," he seems to hold that the brain is the organ by means of which the impressions made on the senses, "the sensational basis of thought," are transmitted to the mind, which then in comparison, judgment, memory, as well as the emotions, acts on the impressions thus received, and that all these mental operations founded on this "sensational basis," take place in the immaterial mind, without any cerebral action or movement. The mind also communicates its volition to the portion of the nervous system connected with the nerves of voluntary movement. In other words, that the mind is placed between the senses which put it in communication with the external world on the one hand and the organs of voluntary movement on the other, and that in all its intermediate intellectual and emotional actions, the brain has no part whatever. If this is the doctrine he holds, it leaves that large portion of the brain between the ganglia of sensation and the apparatus of volun. tary movements entirely without functions; and yet this portion, embracing the whole of the cerebral hemispheres is developed in the proportion of the extent and activity of those same mental operations. It would be easy to show by citations that this view is opposed to 
all the best teachings of modern physiology, and for that reason it would seem unfair to attribute it to Dr. Wilbur, if it were not that the passage occurs in a statement he makes of the greater soundness of the physiological views of the relation of mind to body formerly held, than of those prevailing within the last "twenty years." These peculiar physiological views are in general accordance with the rest of the paper, and furnish a key to the understanding of the "causes, conditions and treatment of insanity" adopted by him.

II.

Application of the Views to the Study of the Causes and Nature of Insanity.

In insanity the mental operations, intellectual, emotional and volitional are disturbed and perverted, and the question arises, does this perversion depend on a change in the entity called mind, or on a structural change in its instrument, the brain?

For those who maintain that the mental operations are only products of cerebral action, that the brain is the mind, no such question can arise. According to their view, a diseased brain gives rise to disordered mental phenomena just as a diseased kidney secretes morbid urine.

So on the other hand, those metaphysicians who shut their eyes to the relation of the mind to the brain, who consider the mental operations as produced by the activity of the entity called mind, without any dependence on the brain, necessarily see in insanity only a disease of the mind.

The question can only arise among those who admit a relation of inter-dependence between the mental operations and the brain. Among these there are some who maintain that although disease of the brain may induce mental derangement, yet that in a certain number of 
cases, mental derangement exists without cerebral disease, or at least pre-exists to it, and constitutes the primary and essential element of the ailment; while others maintain that there is no such thing as disease of the immaterial entity called mind, and that in insanity there is always disease of the cerebral structure, and that the morbid mental manifestations depend on this disease.

It is impossible to establish by adequate scientific evidence either of these hypotheses, for neither is susceptible of direct demonstration, and both explain to a certain extent, the facts of insanity; but the strong tendency of opinion among pathologists is towards the adoption of the latter, as being more completely coincident with facts and more in analogy with physiological and psychological science.

We know that physical disease of the brain originat ing in itself or in other organs often does cause mental derangement. In meningitis or accidental violence, the delirium follows so manifestly the physical lesion that there can be no doubt as to which is cause and which is effect. The same may be said of those transient men. tal derangements caused by medicines or by temporary ill health. The dullness of mind during laborious digestion is plainly due to cerebral change caused by gastric irritation. In the mental torpor and derangement caused by the circulation of diseased blood through the brain, as in uræmia, anæmia and other diseases of this character, the same order of sequence is manifest.

All agree then, that morbid conditions of the brain may cause morbid mental phenomena; but while some hold with Dr. Gray that these material conditions are present in all cases of insanity, others hold with Dr. Wilbur that these material conditions are in many cases 
absent, and that in those cases in which they are found, they are the consequences and not the causes of the insanity.

The cases in which no lesions are found after death in the brain of the insane, are now rare and may be con. sidered exceptional. In proportion as the science of cerebral histology has advanced, and as the means of investigation by the microscope has become more extended, these exceptional cases have become more and more rare, and it may now be fairly concluded that the reason for their occurrence is to be sought in the still imperfect condition of our means of research, or in the want of due care. We may reason here as in the cases of sudden death, in which expert pathologists fail to find any lesion sufficient to account for death, and in which we attribute the failure to the imperfection of science, or want of sufficient care, rather than to a disorder of the vital principle apart from any physical lesions.

It is, however, maintained by those who hold insanity to be a purely mental disease, that even admitting the presence of cerebral lesions in the great majority of cases, or even in all cases of insanity, yet that these lesions are the results or concomitants, and not the causes, of the disordered mental operations.

It may be true that the perversion and violence of the mental operations of the insane give rise to cerebral disease, for this is the conclusion to which our physiological knowledge would lead us. If every mental act is accompanied by some corresponding molecular movement in the brain, it would follow that when these acts are intense and prolonged, the molecular movements will be of like energy, and may result in a permanent change of structure. As a gland, whose functional ac. tion is from any cause perverted or excessive, becomes 
after a time changed in its structure, and then though the cause ceases to operate, this change of structure perpetuates the morbid functional action; so it is conceivable that the brain may become diseased by reason of mental excitement, and continue to exhibit morbid phenomena when the excitement ceases to operate. Thus we may understand how the ravings of an insane man may produce or aggravate disease of the brain, and while I can not admit that a man with a perfectly healthy brain can be insane, yet I would not maintain that a portion of the cerebral lesions found in the.insane after death, may not be due to the insanity itself.

In the study of these phenomena we must keep constantly in view the inter-dependence of the brain and mental operations. Disease of the brain causes disordered mental acts, and these by their violence and irregularity cause disease of the brain, and thus the primary disease is extended and aggravated. Chronic gastritis causes indigestion, and this indigestion leaves the food to ferment in the stomach, aggravating by its presence the gastritis, and thus the consequences of the disease increase the disease. Doctor Gray has pointed out how different forms of insanity pass through dementia in their progress towards recovery. May it not be that the repose of dementia puts a stop to this action of disordered mind in extending and increasing the cerebral disease, leaving this disease to be overcome by natural processes, and acts like abstinence from food in gastritis, relieving the inflamed surface from the irritation of matters it no longer can digest?

Admitting then that mental excitement may influence the cerebral structure and cause disease, it remains to speak of the operation of moral causes in inducing insanity, on which Dr. Wilbur lays great stress. I think

Vor. XXIX.-No. I.-F 
he has herein misapprehended the position taken by Dr. Gray. I do not understand the latter to affirm that moral causes have no agency in the production of insan. ity, but rather that they cause insanity only by causing disease of the brain, and that their agency is not so important as has been supposed. He says, page 11:

"While experience shows that the morbid conditions of organs and tissues more frequently act on the brain than the converse, and thus disease of special organs, and general ill health from lowered vitality, precede and become the cause of the morbid state of the brain, ultimating in insanity; still there are cases where the general ill health and the insanity are due to an overworked brain, or the anxiety and prolonged tension and sleeplessness which are often the result of grief and pecuniary losses. Even here, however, the cause is physical, because insanity comes on only as a result of defective nutrition in the tissues, those of the brain included ; the sleeplessness and deprivation of rest acting powerfully, not only against appetite and the simple ingestion of food, but also by wearying the nerve-tissues, and preventing ultimate cell nutrition. Thus some persons fail suddenly and rapidly, and die unexpectedly. We say these die of exhaustion. But they are not always emaciated, and thus exhausted. The brain gives way, fails in vital energy, and death ensues. Here the morbid action is not in the nature of shock,--of sudden arrest of heartaction by a sudden and powerful impression on the brain,-but of tension and wearing effort, steadily and powerfully depressing the vital energy."

The sequence of events is in this wise. A severe and overwhelming affliction falls upon some person previously sound in mind and body. At once the whole organism is disturbed, the mouth becomes dry and pasty, digestion ceases or is imperfect, the bowels are constipated or relaxed, the action of the heart is tumultuous, the eyes are suffused, and the patient has pain or sense of fullness in the head, with sleeplessness suggest. ing the intense nolecular actions corresponding to the emotional excitement. If this state is prolonged, the 
patient continuing to suffer, his health fails, and in time he may even die of it, remaining perfectly sane. If, however, from some hereditary or other predisposition together with the loss of vitality in the brain caused by the general disturbance, this latter organ becomes diseased, he then is insane. His insanity is then said to arise from a moral cause, and to a certain extent this is true; but it is not till the brain is involved that he is insane, and the occurrence of this brain disease depends not on the degree of mental excitement only, but on other conditions of which I will speak presently.

This may be illustrated by what occurs in other organs. An indigestible meal causes the usual symptoms of gastric irritation, which last till the stomach has got rid of the disturbing cause, and then all the symptoms cease, and the patient is again in health. But if this occurs repeatedly, the stomach becomes changed in structure, and the symptoms persist after the cause bas ceased to operate. This persistent disease of the stomach is analogous to the cerebral disease caused by men. tal excitement, and which is permanent after the moral cause has ceased to exist; for though a removal of the moral cause relieves the distress of mind which precedes insanity, yet, when this latter condition has once been induced, such removal is of little avail.

A person engaged in speculation finds himself embarrassed, and has reason to think that he is reduced to poverty and ruin. This produces great distress of mind; but if his affairs turn out better than he expected so that he finds his fears were groundless, his anxiety is relieved, and his mind and body are again well. If, however, this mental condition had once induced such cerebral disease as to render him insane, an improvement in his affairs has no influence in restoring him to sanity. The reason is that the physical disease once 
established runs its course in spite of the cessation of the moral cause.*

The difference between Dr. Gray and Dr. Wilbur is this: The former as we have seen does not deny the agency of moral causes in producing insanity, but maintains that such causes first induce disease of the brain or of the system generally, and ultimately of the brain, and that when such disease exists the mental operations are perverted or disordered, the patient is insane. Dr. Wilbur maintains that moral causes induce a change in the immaterial entity mind, by which independently of any cerebral disease, its actions are disordered and insanity exists.

If with Dr. Wilbur we regard the brain as only "the organ through which sensational basis of thought" is received, leaving all emotional and intellectual processes independent of the brain, then his view of the operation of moral causes is sufficiently plausible. On the other hand, if we adopt the physiological views advocated by Dr. Gray, and which are sustained by experi. mental and comparative physiology, holding that no emotional or intellectual act goes on without a cerebral movement, the dependence of insanity on physical disease follows as a natural consequence.

We now come to another point on which the Doctors differ, and that is in relation to the importance of moral causes in the production of insanity. Dr. Wilbur holds them to be primary and most frequent. Dr. Gray considers them secondary and comparatively unimportant.

* And this presents a fair illustration of the inefficacy of some forms of moral treatment of insanity. You may explain to this man when insane, that his apprehensions had been erroneous, that his affairs are in fact prosperous, and all this will avail nothing to his recovery. Only when the physical injury has been repaired by time and treatment will sanity return. 
Pathologists have admitted a certain class of diseases, in which are included gout, scrofula, tuberculosis, cancer, insanity and others, which make their appearance at certain periods of life and which depend for their production, not on external conditions, but on certain innate peculiarities of constitution. If we would look for their cause, we must go back to the germ at the moment of fecundation, or even to conditions existing in the parents. This cause is of the same nature as that which determines the conformation of the body, the color of the hair, or the characteristic, intellectual, or moral peculiarities. These are called diseases of development.

Accordingly, we find that a person born of gouty parents, begins at a certain period of life to present symptoms of gout, coming on without any external cause, or if some cause favoring its production is present, it is plainly secondary-no modification of external conditions will altogether prevent the occurrence of the disease in one constitutionally subject to it, nor will any possible external conditions produce it in one not thus disposed.

Yet, from the common tendency to look for direct and palpable causes, the appearance of these diseases is commonly ascribed to accidental causes. A paroxysm of gout is ascribed to luxurious living, tubercular phthisis to exposure to cold, cancer to the inflammation following some bruise. In one sense these are real causes; but it ought to be understood that they would not produce the disease if the internal conditions were not previously present, and that the presence of these internal conditions would in many instances have produced the disease without the concurrence of those external causes.

Insanity is a disease of this class, and often appears 
without any apparent cause. A person not exposed to any great anxieties or afflictions, whose affairs are prosperous, and whose general health has been good, begins to complain of general bad health, and soon after exhibits mental peculiarities which at first do not attract much attention. His family and friends are surprised and griered at his conduct, but do not suspect the real character of the disease, which may continue in this stage for some time before it assumes the form of manifest insanity.

Beginning thus without any apparent cause, it after a time creates an apparent cause for itself, leading an inexperienced observer into error. I will illustrate what I mean by a typical example.

A. cashier of a bank who had for years conducted his own affairs and those of the bank prudently and honestly, began to plunge into hazardous and desperate speculations, and in a short time brought himself and his bank to pecuniary ruin. All this, of course, brought on him disgrace and embarrassment, and after a time he began to exhibit manifest symptoms of mental derangement. He was sent to an asylum at which he died of general paresis.

In this case, the insanity was attributed by his friends to the mortification and distress caused by his disastrous operations, when in reality it was the cause and not the effect of these operations. Exaggerated notions of wealth, and an impulse to reckless speculations are symptoms of the early stage of this disease, which originated in an internal condition without external cause, physical or moral, and which gave rise to an apparent cause. A careless observer might have set this down as a case of insanity depending on a moral cause, distress, occasioned by loss of property and character. 
This explains the case of many a bankrupt who has ended his life in an asylum, and of many a suicide who after losing property and character has put an end to his life before his insanity had been recognized. In many of the cases of insanity thus attributed to moral causes, it will be found on careful examination, that those supposed moral causes are themselves the effect of the insanity.

When some great excitement, political, religious, or speculative, pervades a community, a certain number of persons become insane on the subject of the excitement, and the insanity is then said to depend on this moral cause. The true sequence of events is this.

A religious revival, as it is called, exists in a certain community; meetings are held and frequented by persons interested in the movement, and the excitement of all increases the excitement of each one; discourses presenting terrific pictures of sin and its consequences are delivered, prayers are offered, cries are uttered driving this excitement to frenzy. This state of things continues for some days or weeks, and then subsides. The results are, that a large part of those who were the subjects of this excitement go back to their ordinary business forgetting all about it,-no trace has been left in their minds. A certain number calm down into a quiet religious life, and a few become insane. The latter are said to become insane because of religious excitement, when in truth they were just ready to become insane, and the prevailing excitement only started the disease and gave direction to it. Had a political revolution broken out at the time, it would probably have affected the same subjects, and given a different direction to their insane ideas.

In this way great excitements pass over a community, gathering up all the insane subjects, or those in whom 
the insanity is just ready to break out; just as a high wind, passing over a forest, blows down all the rotten trees just ready to fall, and which in a short time would have fallen without it.

We may call these accidental external conditions, causes of insanity, if we will, just as we may call exposure to cold the cause of consumption, or a bruise of the breast the cause of cancer; but if we make this statement without explanation or qualification, it leads to unsound notions of the etiology and pathology of insanity. These are causes, as the pulling of the trig. ger is the cause of the discharge of the gun. It is indeed a cause, but what a number of conditions must exist in order to render this cause operative. These conditions are of more importance in explaining the explosion, than the slight cause which set them in movement; and so, it is of more importance to understand the preliminary physical condition which renders insan. ity possible, than the accidental cause which gives activity to this condition. The illustration here used fails in this, however, that while the gun would never have been discharged if no one had pulled the trigger, the man, having in himself the internal conditions of insan. ity, is exposed in ordinary life to so many exciting causes, that he could scarcely expect to escape for any length of time.

Shall we then say that men become insane by a sort of predestination, and that it is of no avail to avoid what are calied moral causes? Most assuredly not. One disposed to tubercular disease, may, by choice of climate, nourishment, and other external conditions, escape the development of the disease, and live to an old age; and so, one who, from inheritance or other causes, has all the internal conditions of insanity, may, by a proper regimen and intellectual and moral discipline, escape an 
outbreak of the disease. We certainly would not expose such a one to the excitement of Wall street, or the violent agitations of politics. This shows how unimportant is the part played by moral causes in the production of insanity.

It would be rash to attempt to answer positively the question whether a predisposition must always exist in order to give efficacy to the exciting causes of insanity. Some analogies presented by other diseases of that class, would point to an affirmative answer. We know that some become insane from very slight exciting causes, or even from no apparent cause, while others go through the most overwhelming afflictions of body and mind for many years without becoming insane, so that it would seem that some are incapable of becoming insane under any circumstances, and some have no chance of escaping it. Between these two classes are those who may escape by proper care, and these are the subjects for the skill of the psychological hygienist.

What has been said in regard to moral causes of insanity prepares us to appreciate the importance of what is called its moral treatment. Dr. Wilbur dwells on this as one of the points in which he differs from those who maintain that insanity is a physical disease, though a little examination. will show that the difference is not such as he suspects. A judicious physician does not neglect moral influences in the treatment of any disease, and certainly they are not of less importance in mental derangement depending on cerebral lesion than in other forms of disease. No one disputes the good influence of a placid and hopeful condition of mind in a case of pneumonia or rheumatism or any other purely physical disease. Firmness and gentleness on the part of attendants, avoidance of all violence and excitement, such sur. roundings as may induce in the patient a cheerful and 
placid temper, together with a healthy exercise of the moral and intellectual faculties are remedial and hygienic measures, which no wise physician would question or discard in the management of insanity, however much he might be convinced of the physical character of the disease. If we admit that insane thoughts and acts have a tendency to aggravate and extend the cerebral lesion, it is obvious that an important element of treatment is to allay the violence of the mental operations by soothing moral treatment. Indeed, it is worthy of remark, that the adoption of such treatment dates from the time that the insane began to be considered as sub. jects of physical disease and not victims of demoniacal possessions.

So far, then, there can be no dispute as to the necessity of moral treatment; it accords quite as well with the pathological views of Dr. Gray as with the views of Dr. Wilbur. But, if we attempt to operate directly on the mind of the insane by moral considerations we shall be greatly disappointed in the results. The importance of moral treatment lies in avoiding what is hurtful, but it is of little influence as a direct curative agent. How vain is the attempt to reason with the insane on the subject of their delusions, or to remove the gloom and despondency of melancholics by cheerful or diverting conversation is well understood.

This may be illustrated by what occurs in the experience and consciousness of most persons, in a condition, not of insanity, but presenting similar mental and physical elements.

Most persons know what it is to be in low spirits without any external cause to justify or explain the feeling. In this state of miserable despondency everything looks gloomy and threatening. The attempt of friends to cheer the subject of this sadness and appre- 
hension, by showing how unreasonable it is, or by diversions and cheerful conversation, only excites a feeling of vexation and anger. Now, under such circum. stances, the operation of a cholagogue purgative will often restore the sufferer at once to his accustomed humor and to moral and intellectual activity. The mental disturbance depended on the circulation through the brain of blood imperfectly depurated, and ceases at once when this fluid has been restored to its normal condition. All moral curative treatment failed while the physical cause continued, and became unneccessary when this cause was removed. This condition differs from melancholia in being of a slight and transient character, while the latter depends on a more deeply rooted and permanent physical lesion. Melancholics who have recovered, describe the passing away of their delusions and sadness as being like the dispersion of a cloud which had been hanging over them.

In this paper the attempt has been made to present fairly the spirit and matter of the views and arguments on both sides, without introducing extracts from the papers which have served as the text. Such extracts would have extended this paper to an unreasonable length, and were unnecessary for the reason that the papers under consideration are very generally in the hands of the readers of this JournaL. 